\title{
BMJ Open Availability and Quality of Assessment Instruments on Patient-Centredness in the Multimorbid Elderly (AQuA-PCE): a study protocol of a systematic review
}

\author{
Manuela Glattacker (D , ${ }^{1}$ Manuela Kanat, ${ }^{1}$ Jonas Schaefer, ${ }^{1}$ Edith Motschall, ${ }^{2}$ \\ Laura Kivelitz, ${ }^{3}$ Sebastian Voigt-Radloff, ${ }^{4}$ Joerg Dirmaier ${ }^{3}$
}

To cite: Glattacker M, Kanat M, Schaefer J, et al. Availability and Quality of Assessment Instruments on Patient-Centredness in the Multimorbid Elderly (AQuAPCE): a study protocol of a systematic review. BMJ Open 2020;10:e033273. doi:10.1136/ bmjopen-2019-033273

- Prepublication history and additional material for this paper are available online. To view these files, please visit the journal online (http://dx.doi org/10.1136/bmjopen-2019033273).

Received 29 July 2019 Revised 18 December 2019 Accepted 06 January 2020

D) Check for updates

(c) Author(s) (or their employer(s)) 2020. Re-use permitted under CC BY-NC. No commercial re-use. See rights and permissions. Published by BMJ.

For numbered affiliations see end of article.

\section{Correspondence to}

Dr Manuela Glattacker;

manuela.glattacker@uniklinikfreiburg.de

\section{ABSTRACT}

Introduction Elderly, multimorbid patients are a primary target group for patient-centred care, and fostering patient-centredness $(\mathrm{PC})$ in this group has been associated with different healthcare aims such as safety and quality of healthcare. However, evidence on effects of patientcentred interventions is still limited and mixed. In part, the lack of consistent evidence has its roots in a conceptual uncertainty of the term 'PC', which also hampers the development of assessment tools for PC. Consequently, reviews on assessment instruments of $\mathrm{PC}$ reveal problems regarding the quality of identified assessment instruments and regarding their comparability. Some of these reviews focus on the elderly. However, while the concept of multimorbidity is partly inherent, this focus is not explicit in any of the reviews.

The aim of this systematic review is to identify assessment instruments of PC in the multimorbid elderly, using a subgroup-specific definition of PC ('subgroup-specific integrative model of $\mathrm{PC}^{\prime}$ ) as the conceptual underpinning, and to provide a critical quality appraisal of their psychometric properties.

Methods and analysis A comprehensive systematic literature search for assessment tools on PC will be conducted in the MEDLINE, CINAHL, EMBASE, PsycINFO, Web of Science and PSYNDEX electronic databases. The search strategy will be informed by the subgroupspecific integrative model of PC. The electronic literature search will be complemented by a hand-search combining citation tracking, search in project databases, and contacting authors from relevant studies/reviews. The literature search (systematic and hand-search) will cover the period from November, 2018 to December 2019.

The retrieval of relevant studies will be conducted via title screening, abstract screening, and full-text eligibility assessment applying defined inclusion criteria. Full texts will be independently assessed by two team members. Data from the included articles will be extracted using a standardised extraction form and evaluated based on the COSMIN methodology for systematic reviews of patient-reported outcome measures, which focuses on the methodological quality of included studies as well as on the measurement properties of the assessment instruments. Data extraction and quality assessment will be conducted by two independent reviewers.
Strengths and limitations of this study

- The systematic review is the first to identify assessment instruments of patient centredness (PC) explicitly for the multimorbid elderly.

- The systematic review considers a subgroupspecific adaptation of the 'integrative model of PC' as the conceptual underpinning, therefore including the perceptions of the target group of the multimorbid elderly on PC.

- The search strategy combines commonly used terms for PC (such as 'patient centredness') with search terms for the four dimensions of the subgroup-specific integrative model of $\mathrm{PC}$, which have been prioritised for the multimorbid elderly by experts in a prestudy, namely 'patient involvement in care', 'clinician-patient communication', 'physical and emotional support' and 'involvement of family and friends'

- The review applies quality criteria for systematic reviews on assessment instruments as suggested by the COSMIN guideline.

- Tailoring the search strategy to the four abovementioned prioritised dimensions of the subgroupspecific integrative model of PC can be considered a limitation of the review.

Ethics and dissemination The study has received approval from the Ethics Committee of the University of Freiburg (reference number 587/17). The results of the project will be disseminated via scientific oral presentations at national and international conferences and will be published in scientific journals.

Trial registration numbers CRD42018084057; DRKS00013309.

\section{INTRODUCTION}

Patient-centredness (PC), defined as the individualisation of healthcare processes ${ }^{1}$ by considering the specific needs, preferences and values of the individual patient, has been pointed out as a key dimension of high-quality healthcare ${ }^{2}$ and is in the focus of many public 
healthcare programmes worldwide. According to the $\mathrm{WHO},{ }^{3}$ a patient or people-centred approach in healthcare is needed to ensure equity in access, quality, responsiveness and participation, to increase the efficiency of healthcare interventions and the resilience against potential public health crises. ${ }^{3}$

Elderly, multimorbid patients have been identified as a primary target group for patient-centred care. ${ }^{4}$ Due to the demographic trend of ageing populations, the number of elderly patients (65 years and older) is expected to continue to increase, ${ }^{5}$ with more than half of the older population suffering from multimorbidity (defined by the presence of multiple chronic conditions) ${ }^{67}$ In old age, multimorbidity is considered to be particularly complex due to the overlap of physical and mental health disorders, frailty and polypharmacy. ${ }^{8}$ Therefore, elderly, multimorbid patients have complex healthcare needs which often largely differ from the needs of younger patients. ${ }^{9-13}$ These must be taken into account when thinking about patient-centred care for this target population.

Fostering PC in the care of older adults with multimorbidity has been associated with different healthcare aims and outcomes such as coordination, safety and quality of healthcare, as well as quality of life. ${ }^{14}$ Evidence suggests that PC indeed has an impact on different health related outcomes $^{1516}$ and might decrease healthcare utilisation and medical care charges. ${ }^{1718}$ However, Cochrane Reviews of PC interventions evaluate the existing evidence on the effects of patient-centred interventions on patient satisfaction, health behaviour and health status as still limited and mixed. ${ }^{1}$

In part, the lack of consistent evidence has its roots in a conceptual uncertainty of the term 'patient-centredness', which is reflected in different and only partly overlapping definitions of PC. ${ }^{16-21}$ With the goal to clarify the conceptual base of $\mathrm{PC}$, our research group systematically reviewed existing definitions of PC. Based on 417 articles containing a definition of $\mathrm{PC}$, we proposed the 'integrative model of PC' comprising 15 different dimensions: essential characteristics of clinician, clinician-patient relationship, clinician-patient communication, patient as a unique person, biopsychosocial perspective, patient information, patient involvement in care, involvement of family and friends, patient empowerment, physical support, emotional support, integration of medical and non-medical care, teamwork and teambuilding, access to care, coordination, and continuity of care. ${ }^{22}$ The model was validated in a Delphi study conducted with international experts ${ }^{23}$ and is actually evaluated from the patients' perspective. ${ }^{24}$ Furthermore, the model has been refined to fit elderly multimorbid patients (henceforth referred to as 'subgroup-specific integrative model') using a DelphiStudy with $n=48$ experts ${ }^{25}$ and focus groups with $n=20$ multimorbid geriatric patients (13 male, 7 female) aged between 69 and 90 years, who suffered from two or more chronic medical conditions mostly relating to the circulatory system, the musculoskeletal system or the nervous system. ${ }^{26}$ Adapting some dimensions for the specific needs of the multimorbid elderly, the overall structure of the model has been validated, and the experts prioritised the following dimensions as most important for the multimorbid elderly: 'patient involvement in care', 'clinicianpatient communication', 'physical and emotional support', and 'involvement of family and friends'. ${ }^{25}$

Prospectively, the integrative model of PC might facilitate the development of related assessment instruments and interventions. To date, however, the long-prevailing conceptual uncertainty of the term PC hampers the comparison and quality assessment of existing assessment tools, which can be concluded from several reviews of assessment instruments of PC. For example, Hudon $e t a l^{27}$ reviewed tools regarding patients' perceptions of patientcentred care in family medicine. Searching for different terms of PC (such as 'patient-centred care', 'patient focused care' or 'patient centredness') they identified two instruments measuring patient-centred care and some subscales or single items from other instruments providing only partial coverage of the concept PC.

Köberich and Farin ${ }^{28}$ reviewed instruments measuring PC within the nursing setting and evaluated their psychometric properties. Using different search terms for PC (such as patient/person/client/resident-centred/ focused care, personalised/individualised/tailored care/ nursing), the authors found four instruments, which predominantly covered themes addressing patient participation and the clinician-patient relationship. Although lacking information regarding some psychometric properties, the quality of these instruments was interpreted to be satisfactory.

There are also some reviews explicitly focusing on the measurement of PC for the elderly. Edvardsson and Innes $^{29}$ reviewed instruments measuring the PC of care for older people and people with dementia. Applying a search strategy that used various combinations of the terms 'person-centred', 'patient-centred' and 'individualised' paired with 'Alzheimer's disease', 'older people' and 'dementia', the review identified 12 tools. However, none of the instruments have been used and validated beyond the development period. Kogan $e t a l^{30}$ identified 13 tools measuring PC in healthcare-related settings using the search terms 'person-centred care' and 'patient-centred care' individually and each combined with 'older adults' or 'elderly'. However, 10 of these tools have not been tested for validity or reliability. Furthermore, Wilberforce et $a \hat{l}^{31}$ conducted a systematic review of questionnairebased scales and their measurement properties assessing person-centredness in the care of older adults. Using the terms 'person-centred' (including the prefixes 'patient', 'consumer', 'client' and the suffixes 'led', 'oriented', 'directed' to 'centredness') and also including 'individualised' and 'personalised' alternatives as search terms for PC, they identified 11 assessment instruments. However, none of the instruments were suitable to fit all of the determined measurement properties resulting in the conclusion that the methodological quality of measurements needed to be improved. 
Taken together, all of these reviews revealed some problems regarding the quality of the assessment instruments and regarding their comparability due to the conceptual uncertainty of the underlying concept of PC. Furthermore, while the concept of multimorbidity is partly inherent in the above-mentioned reviews focusing on the elderly, this focus is not explicit in any of the reviews.

\section{Objectives}

This systematic review aims to identify existing assessment instruments of PC in the multimorbid elderly and to provide a critical quality appraisal of their psychometric properties. As a conceptual underpinning of PC, we apply the above-mentioned subgroup-specific comprehensive integrative model of PC. ${ }^{22} 2526$

\section{METHODS AND ANALYSIS}

In designing and reporting this study, we followed the PRISMA guideline for systematic reviews. ${ }^{32}$ Furthermore, we applied the recommendations of the COSMIN group (https://www.cosmin.nl/). The study was registered within PROSPERO (https://www.crd.york.ac.uk/prospero; CRD42018084057; 2018/02/01) and the German Clinical Trials Register (www.drks.de; DRKS00013309; 2018/01/23).

\section{Literature search and study selection}

Systematic searches will be conducted in the MEDLINE, CINAHL, EMBASE, PsycINFO, Web of Science and PSYNDEX electronic databases to identify relevant articles published up to 6.11.2018 (these databases slightly differ from those listed in PROSPERO: We will use the search interface Ovid provided by Wolters Kluwer as it provides more options as compared with Pubmed (eg, proximity search)). Separate search strategies were set up to identify citations in the MEDLINE databases for indexed and non-indexed citations. The search terms and the search strategy were developed with the support of an experienced librarian (EM). The search strategy combines different search terms for the key search components 'patient centredness', 'elderly/age', 'assessment tool' and 'multimorbidity'. With respect to 'patient centredness', we derived different terms from previous reviews on $\mathrm{PC},{ }^{1} 3031$ such as 'patient/person/client/ consumer/people/resident' combined with 'centred/ centered/oriented/directed/focused'. Furthermore, we defined search terms for the four dimensions of the integrative model of PC which had been prioritised for the multimorbid elderly in the preceding Delphi survey: 'patient involvement in care', 'clinician-patient communication', 'physical and emotional support' and 'involvement of family and friends' (see above). For the search component 'assessment instrument' we modified existing filters proposed by Terwee $e t a l .{ }^{33}$ For the search component 'elderly/age', we considered the Ovid filter 'Elderly' for EMBASE together with the associated search filters for geriatric medicine proposed by van de Glind $e t$ $a l,{ }^{34}$ and modified these filters to fit our needs. For the search term 'multimorbidity', we included terms which were selected from an ongoing project on patient preferences in multimorbid patients ${ }^{35}$ and from a Cochrane review on interventions in multimorbid patients, ${ }^{36}$ and we complemented these terms with indicators for multimorbidity such as polypharmacy (see online supplementary appendix 1 for the specific search strategy in MEDLINE as an example).

The language of publication was restricted to English or German. Further limiters with respect to publication types and study types were defined specifically for each database. For example, for MEDLINE, the following publication types were excluded from our search: address, biography, case report, comment, directory, editorial, festschrift, interview, lecture, legal case, legislation, letter, news, newspaper article, patient education handout, popular works, congress, consensus development conference or practice guideline. With respect to study types, we excluded studies that were explicitly indexed as animal studies or 'not humans'. However, as experimental studies/RCTs (Randomized Controlled Studies) may incorporate the validation of an assessment instrument, we did not exclude this study type from our search strategy.

In addition to the systematic electronic search, we will conduct a hand-search that will combine different approaches: In the context of reference tracking, we will hand-search reference lists of studies included in our review for further publications relevant to that topic. Furthermore, we will search PROSPERO for ongoing or unpublished systematic reviews on the topic of interest, and we will contact first authors of relevant reviews and studies in order to identify further articles. The literature search (systematic and hand-search) will cover the period from November 2018 to December 2019.

The retrieval of relevant studies will be conducted sequentially via title screening, abstract screening and full text eligibility assessment. Inclusion criteria for the selection of the studies are:

1. An assessment instrument of PC or its dimensions (as defined within the subgroup-specific integrative model of PC) is mentioned within the article. In order to capture different perspectives, we consider self-report questionnaires as well as external assessments (by professionals or relatives) and observation-based measures as 'assessment instruments'.

2. The article reports psychometric properties of the assessment instrument.

3. The assessment instrument was developed or reported for our target group (the multimorbid elderly).

4. The language of the publication and assessment instrument is English or German.

5. The article is published in a peer-reviewed journal.

6. The full text of the article is available.

Due to feasibility reasons, title and abstract screening will be conducted by one team member. However, in a sample of $5 \%$ of the data set, two authors will independently 
screen title and abstracts to test if the screening strategy is reasonable. Furthermore, the team members will be in close contact to resolve any problems or questions during the title and abstract screening process. Full text eligibility assessment will then be conducted independently by two team members, and disagreements will be reconciled promptly throughout the screening processes. The results of the identification, screening and inclusion process will be displayed using the PRISMA flowchart. ${ }^{32}$ The management of records and data will be done via Rayyan. ${ }^{37}$

\section{Data extraction and quality assessment}

The data from the included articles will be extracted using a standardised extraction form and evaluated oriented towards the COSMIN methodology for systematic reviews of patient-reported outcome measures (PROMs), which supplements the COSMIN guideline for systematic reviews of measurement instruments ${ }^{38}$ by including the COSMIN Risk of Bias checklist. ${ }^{39}$ The Risk of Bias checklist is used for assessing the methodological quality of the studies included in the systematic review.

The COSMIN methodology suggests the following procedure: ${ }^{38-40}$ After the literature search, the methodological quality of each study reporting on a measurement property is assessed using the COSMIN Risk of Bias checklist. In this context, it is first determined which measurement properties are assessed in each article. The COSMIN Risk of Bias checklist is used as a modular tool, completing the boxes of the checklist only for the measurement properties evaluated in the article. The quality of each study is rated as 'very good', 'adequate', 'doubtful' or 'inadequate' applying standards which refer to design requirements and preferred statistical methods for evaluating the quality of studies on measurement properties.

Second, data are extracted regarding the characteristics of the PROM, the characteristics of the included study population and the results on the measurement properties. The results of each study are then evaluated against criteria for good measurement properties as either sufficient $(+)$, insufficient $(-)$ or indeterminate (?) (eg, for internal consistency, '+' means 'at least low evidence for sufficient structural validity AND Cronbach's alpha(s) $\geq 0.70$ for each unidimensional scale or subscale', while '-' means 'at least low evidence for sufficient structural validity AND Cronbach's alpha(s) $<0.70$ for each unidimensional scale or subscale'). The following measurement properties of the included PROMs are evaluated: content validity, ${ }^{40}$ structural validity, internal consistency, cross-cultural validity/measurement invariance, reliability, measurement error, criterion validity, hypothesis testing for construct validity and responsiveness.

Third, the evidence is summarised for each measurement property of each PROM, the overall result is rated against criteria for good measurement properties, and the quality of the evidence is graded by using a modified GRADE approach, which grades the quality of the evidence as high, moderate, low or very low.

This procedure will be adapted for our purposes including the adaptation of the process of extraction and evaluation for external assessments and observationbased measures. As the integrative model of PC was validated 'as a whole' for the elderly, we do not give more weight to the adapted dimensions but instead weight the included dimensions equally in the context of the extracting process.

Before starting the final data extraction, a pilot assessment will be conducted for a random sample of approximately $10 \%$ of the included papers in order to improve rater agreement in using the COSMIN checklist. In case of any disagreements, they will be resolved through discussion prior to extracting and rating the data for the remaining $90 \%$ of the studies. Data extraction and quality assessment will be conducted by two independent reviewers.

\section{Patient and public involvement}

Patients were neither involved in the development of the research question nor in the study design. However, the systematic review bases on the subgroup-specific comprehensive integrative model of PC which was adapted through an expert-based Delphi survey ${ }^{25}$ and through focus groups with $\mathrm{n}=20$ multimorbid geriatric patients. ${ }^{26}$ The results of the focus groups have been included in the refinement of the integrative model. Therefore, the perceptions of PC from the perspective of the multimorbid elderly as well as their specific needs and values regarding $\mathrm{PC}$ have been incorporated in the search strategy of this review.

Additionally, we plan to publish the study results in plain language and to disseminate these plain language results to study participants as well as treatment facilities which are involved in the care of the multimorbid elderly (eg, the Center for Geriatric Medicine and Gerontology at the University of Freiburg).

The public will be informed of the study and its results through the website of our department (https://www. imbi.uni-freiburg.de/SEVERA/Projekte/aktuell/aqua) and through the publication of project descriptions in newsletters of our department.

\section{ETHICS AND DISSEMINATION}

For the systematic literature review in the narrow sense, several ethical and safety considerations such as informed consent, confidentiality requirements or data protection guidelines do not apply. However, the study as a whole, which contains the systematic literature review presented here, is subject to the above-mentioned requirements and has received approval from the Ethics Committee of the University of Freiburg (reference number 587/17).

Concerning the dissemination plan, for one we will inform our collaboration partners and the interested public about the project using the newsletter of our 
department. Furthermore, the results of the project will be disseminated via scientific oral presentations at national and international conferences and will be published in scientific journals. If feasible, we will choose open access journals in order to ensure high accessibility to the study results.

\section{DATE OF THE STUDY}

The whole study, which also included a Delphi survey and patient focus groups (not considered within this study protocol) started on 1 September 2017. The systematic review, which is part of the study, started on 1 September 2018 and will be finished on 31 December 2019. The final report will be due on 30 June 2020 .

\section{Author affiliations}

${ }^{1}$ Section of Health Care Research and Rehabilitation Research, Faculty of Medicine and Medical Center-University of Freiburg, Freiburg, Germany

${ }^{2}$ Institute for Medical Biometry and Statistics, Faculty of Medicine and Medical Center-University of Freiburg, Freiburg, Germany

${ }^{3}$ Department of Medical Psychology, University Medical Center Hamburg-Eppendorf, Hamburg, Germany

${ }^{4}$ Center for Geriatric Medicine and Gerontology Freiburg, Institute for Evidence in Medicine (for Cochrane Germany Foundation), Faculty of Medicine and Medical Center-University of Freiburg, Freiburg, Germany

Acknowledgements We thank Andrea Feigel for proofreading and her critical review of the manuscript. The article processing charge was funded by the German Research Foundation (DFG) and the Albert-Ludwigs-University of Freiburg in the funding programme Open Access Publishing.

Contributors MG has made substantial contribution to the conception of the systematic review and was a major contributor in writing the manuscript. MK, JS, EM and LK have made substantial contribution to the concrete elaboration of the systematic review predominantly in terms of the search strategy and was a contributor in writing the manuscript. SVR has made contribution to the conception of the systematic review and contributed in writing the manuscript. JD has made contribution to the conception of the systematic review and was a contributor in writing the manuscript. All authors read and approved the final manuscript.

Funding The study is funded by the Bundesministerium für Bildung und Forschung (01GL1724).

Competing interests None declared.

Patient consent for publication Not required.

Ethics approval The study has received approval from the Ethics Committee of the University of Freiburg (reference number 587/17).

Provenance and peer review Not commissioned; externally peer reviewed.

Data availability statement No data are available. There are no data as this is a study protocol of a systematic review.

Open access This is an open access article distributed in accordance with the Creative Commons Attribution Non Commercial (CC BY-NC 4.0) license, which permits others to distribute, remix, adapt, build upon this work non-commercially, and license their derivative works on different terms, provided the original work is properly cited, appropriate credit is given, any changes made indicated, and the use is non-commercial. See: http://creativecommons.org/licenses/by-nc/4.0/.

ORCID iD

Manuela Glattacker http://orcid.org/0000-0003-4300-2201

\section{REFERENCES}

1 Dwamena F, Holmes-Rovner M, Gaulden CM, et al. Interventions for providers to promote a patient-centred approach in clinical consultations. Cochrane Database Syst Rev 2012;12:CD003267.
2 Institute of Medicine. Crossing the Quality Chasm - A new Health System for the 21st Century. 1st edn. Washington, DC: National Academy Press, 2001.

3 WHO. WHO Framework on integrated people-centred health services. Available: http://www.who.int/servicedeliverysafety/areas/ people-centred-care/en/ [Accessed 8 Sep 2016].

4 American Geriatrics Society Expert Panel on the Care of Older Adults with Multimorbidity. Patient-Centered care for older adults with multiple chronic conditions: a stepwise approach from the American geriatrics Society. J Am Geriatr Soc 2012;60:1957-68.

5 Oeppen J, Vaupel JW. Demography: enhanced: broken limits to life expectancy. Science 2002;296:1029-31.

6 Barnett K, Mercer SW, Norbury M, et al. Epidemiology of multimorbidity and implications for health care, research, and medical education: a cross-sectional study. The Lancet 2012;380:37-43.

7 Salive ME. Multimorbidity in older adults. Epidemiol Rev 2013;35:75-83.

8 Yarnall AJ, Sayer AA, Clegg A, et al. New horizons in multimorbidity in older adults. Age Ageing 2017;46:882-8.

9 Chi WC, Wolff J, Greer R, et al. Multimorbidity and decision-making preferences among older adults. Ann Fam Med 2017;15:546-51.

10 Chiu C, Feuz MA, McMahan RD, et al. "Doctor, Make My Decisions": Decision Control Preferences, Advance Care Planning, and Satisfaction With Communication Among Diverse Older Adults. $J$ Pain Symptom Manage 2016;51:33-40.

11 Farin E, Schmidt E, Gramm L. Predictors of communication preferences in patients with chronic low back pain. Patient Prefer Adherence 2013;7:1117-27.

12 Lechner S, Herzog W, Boehlen F, et al. Control preferences in treatment decisions among older adults - results of a large population-based study. J Psychosom Res 2016;86:28-33.

13 Parker SG, Corner L, Laing K, et al. Priorities for research in multiple conditions in later life (multi-morbidity): findings from a James Lind alliance priority setting partnership. Age Ageing 2019;48:401-6.

14 The American Geriatrics Society Expert Panel on Person-Centered Care. Person-Centered care: a definition and essential elements. $J$ Am Geriatr Soc 2016;64:15-18.

15 Rathert C, Wyrwich MD, Boren SA, et al. Patient-Centered care and outcomes: a systematic review of the literature. Med Care Res Rev 2013;70:351-79.

16 Robinson JH, Callister LC, Berry JA, et al. Patient-Centered care and adherence: definitions and applications to improve outcomes. J Am Acad Nurse Pract 2008;20:600-7.

17 Bertakis KD, Azari R. Determinants and outcomes of patientcentered care. Patient Educ Couns 2011;85:46-52.

18 Bertakis KD, Azari R. Patient-Centered care is associated with decreased health care utilization. J Am Board Fam Med 2011;24:229-39.

19 Epstein RM, Franks P, Fiscella K, et al. Measuring patient-centered communication in Patient-Physician consultations: theoretical and practical issues. Soc Sci Med 2005;61:1516-28.

20 Mead N, Bower P. Patient-centredness: a conceptual framework and review of the empirical literature. Soc Sci Med 2000;51:1087-110.

21 van Dulmen S. Patient-centredness. Patient Educ Couns 2003;51:195-6.

22 Scholl I, Zill JM, Härter M, et al. An integrative model of PatientCenteredness - a systematic review and concept analysis. PLoS One 2014;9:e107828.

23 Zill JM, Scholl I, Härter M, et al. Which Dimensions of PatientCenteredness Matter? - Results of a Web-Based Expert Delphi Survey. PLoS One 2015;10:e0141978.

24 Christalle E, Zeh S, Hahlweg P, et al. Assessment of patient centredness through patient-reported experience measures (ASPIRED): protocol of a mixed-methods study. BMJ Open 2018;8:e025896.

25 Kivelitz L, Kanat M, Schäfer J, et al. Dimensions of patientcenteredness in the multimorbid elderly: results of a web-based expert Delphi survey. In Press.

26 Kanat M, Schäfer J, Kivelitz L, et al. Patient-centeredness in the multimorbid elderly: a focus group study. In Press.

27 Hudon C, Fortin M, Haggerty JL, et al. Measuring patients' perceptions of patient-centered care: a systematic review of tools for family medicine. Ann Fam Med 2011;9:155-64.

28 Köberich S, Farin E. A systematic review of instruments measuring patients' perceptions of patient-centred nursing care. Nurs Inq 2015;22:106-20.

29 Edvardsson D, Innes A. Measuring Person-centered care: a critical comparative review of published tools. Gerontologist 2010;50:834-46. 
30 Kogan AC, Wilber K, Mosqueda L. Person-Centered care for older adults with chronic conditions and functional impairment: a systematic literature review. J Am Geriatr Soc 2016;64:e1-7.

31 Wilberforce M, Challis D, Davies L, et al. Person-centredness in the care of older adults: a systematic review of questionnaire-based scales and their measurement properties. BMC Geriatr 2016;16.

32 Moher D, Shamseer L, Clarke M, et al. Preferred reporting items for systematic review and meta-analysis protocols (PRISMA-P) 2015 statement. Syst Rev 2015;4:1.

33 Terwee CB, Jansma EP, Riphagen II, et al. Development of a methodological PubMed search filter for finding studies on measurement properties of measurement instruments. Qual Life Res 2009;18:1115-23.

34 van de Glind EMM, van Munster BC, Spijker R, et al. Search filters to identify geriatric medicine in MEDLINE. J Am Med Inform Assoc 2012;19:468-72.
35 Muth C, Aksamit N, Von Büdingen F, et al. Patientenpräferenzen bei Multimorbidität und Multimedikation - Pilotierung einer evidence map. Kongr Für Allg Fam 2015.

36 Smith SM, Wallace E, O'Dowd T, et al. Interventions for improving outcomes in patients with multimorbidity in primary care and community settings. Cochrane Database Syst Rev 2016;9.

37 Ouzzani M, Hammady H, Fedorowicz Z, et al. Rayyan-a web and mobile APP for systematic reviews. Syst Rev 2016;5:210.

38 Prinsen CAC, Mokkink LB, Bouter LM, et al. COSMIN guideline for systematic reviews of patient-reported outcome measures. Qual Life Res 2018;27:1147-57.

39 Mokkink LB, de Vet HCW, Prinsen CAC, et al. COSMIN risk of bias checklist for systematic reviews of patient-reported outcome measures. Qual Life Res 2018;27:1171-9.

40 Terwee CB, Prinsen CAC, Chiarotto A, et al. COSMIN methodology for evaluating the content validity of patient-reported outcome measures: a Delphi study. Qual Life Res 2018;27:1159-70. 